\title{
Häufige und schwere Fehler in der Dermatologie
}

\begin{abstract}
Aus den meisten Missgeschicken, die in der Dermatologie passieren, trägt der Patient glücklicherweise keinen Schaden davon. Eine US-Studie sollte aufdecken, welche Fehler sich im praktischen Alltag ereignen. Als besonders kritisch erwiesen sich dabei die lange Handlungskette bei einer Biopsie, die Wahl der richtigen Körperseite bei chirurgischen Eingriffen sowie die medikamentöse Therapie.
\end{abstract}

Auf drei verschiedenen Kongressen wurden amerikanische Klinikdermatologen anonym nach Art und Häufigkeit der Fehler befragt, denen sie in jüngster Vergangenheit in ihrem Arbeitsumfeld begegnet waren. Darüber hinaus sollten sie den schwersten Fehler und seine Folgen beschreiben. Die insgesamt 150 teilnehmenden Ärzte, von denen $60 \%$ älter als 50 Jahre waren, gaben 152 kürzlich erfolgte und 130 folgenschwere Fehler zu Protokoll. Nur zwei Teilnehmer arbeiteten offenbar in einer perfekten Umgebung.

\section{Welche Pannen sind häufig?}

85\% der genannten Pannen hatten sich höchstens einmal im Jahr ereignet und in den allermeisten Fällen (86\%) waren dadurch keine Patienten zu Schaden gekommen. 41\% der Fehler, mit denen die Dermatologen erst kürzlich konfrontiert worden waren, standen im Zusammenhang mit Untersuchung oder Diagnose, 44\% ereigneten sich bei der Behandlung. Dabei fiel auf: Neun von zehn der Untersuchungs- und Diagnostikfehler betrafen Biopsien. Im Bereich Behandlung ging etwas mehr als die Hälfte der Ausrutscher auf das Konto der medikamentösen Therapie. Die Fehler bei anderen Maßnahmen bezogen sich zu rund zwei Dritteln auf operative Eingriffe, das restliche Drittel ereignete sich bei der Phototherapie und bei Laserbehandlungen. Als mit Abstand häufigste Fehlerquelle (18\%) gaben die Ärzte eine falsche Beschriftung auf dem Probengefäß bzw. eine Fehlinformation auf dem Begleitformular an. Mit immerhin 3\% schlug die verwechselte Körperseite bei einem chirurgischen Eingriff zu Buche.

\section{Schwere Fehler sind zum Glück selten}

Knapp ein Drittel der schwerwiegenden Fehler stand im Zusammenhang mit einer Untersuchung oder Diagnosestellung. So kam es beispielsweise zur Bildung von Metastasen, weil die Diagnose eines Melanoms zu spät gestellt wurde. Etwa jeder zweite schwere Irrtum war ein Behandlungsfehler. $73 \%$ davon ereigneten sich bei chirurgischen Eingriffen, in 27\% der Fälle war die Medikation fehlerhaft, insbesondere durch Verschreibungen trotz bekannter Allergien oder Kontraindikationen.

Auf den drei vordersten Plätzen in der Liste der schwerwiegendsten Fehler rangierte die verwechselte Op.-Seite mit 19\% ganz oben, gefolgt von einer falschen klinischen Beurteilung, die zu einer Fehldiagnose führte (14\%). Den dritten Rang nahm die Phototherapie mit $10 \%$ ein, die zum Teil schwere Verbrennungen nach sich zog.

\section{Verstärkte Sicherheitsmaßnahmen gefordert}

Angesichts der vielen Fehler fordern die Autoren verstärkte Sicherheitsmaßnahmen. Dies betrifft unter anderem den gesamten Vorgang einer Biopsie, bei dem von der Probengewinnung bis zum Befund mehr als 20 Übergaben zwischen verschiedenen Personen und Einrichtungen stattfinden können. Hier müsse etwas unternommen werden, um Fehlerquellen aufzudecken. Dem Problem der Seitenverwechslung könne man beispielsweise mit Fotoaufnahmen im Vorfeld einer Biopsie entgegenwirken, meinen die Autoren. Fehler bei der Medikation könnten vermieden werden, indem man sich auf bewährte Verfahren der Verordnung und Überwachung stütze und sich an Leitlinien orientiere. Um die Versorgungsqualität der Patienten zu verbessern, so die Autoren, sollten mithilfe einfacher Bewertungssysteme so viele Fehler wie möglich erfasst und zusammengetragen werden. (ChristineStarostzik)

Watson AJ et al, American Academy of Dermatology 2013 (online first) 